There was a lot of confusion surrounding the use of personal protection equipment and visitation allowances due to this occurring in the early stages of the coronavirus pandemic. This in itself delayed transfer to more suitable wards and added undue stress to an already complicated case. Nevertheless, all staff involved were professional and addressed concerns appropriately to make sure this woman was managed safely and within protocol.

\section{LEARNING POINTS}

- This is a typical presentation of a patient with OS with the added complication of HAP and lower lobe collapse.

- Multiple teams were needed to ensure optimum management especially due to the multiple postoperative complications demonstrated in this patient.

- Extra precautions were needed due to protocols put in place during the coronavirus pandemic.

- Communication was essential in this case to ensure all information was conveyed to the patient and the plan surrounding her care was explained.

Correspondence to: leah.hawkins@mbht.nhs.uk sunday.ajayi@mbht.nhs.uk

\section{REFERENCES}

1. Newson, L. (2015). Ogilvie's Syndrome. Patient UK. Available at: https://patient.info/doctor/ogilvies-syndrome (accessed 11.05.2020).

2. Jayaram P, Mohan M, Lindow S, Konje J. Postpartum acute colonic pseudo-obstruction (Ogilvie's Syndrome): a systematic review of case reports and case series. Eur J Obstet Gynecol Reprod Biol. 2017;214:145-149. Available at:https://www.ncbi.nlm.nih.gov/pubmed/28531835 (accessed 11.05.2020).

3. Hughes A, Smart NJ, Daniels IR. Acute colonic pseudo-obstruction after caesarean section: a review and recommended management algorithm. The Obstetrician \& Gynaecologist, 2019;21(4):pp.283-290.

4. Maloney N, Vargas HD. Acute intestinal pseudo-obstruction (Ogilvie's syndrome). Clinics in colon and rectal surgery, 2005;18(2):96-101. Available at: https://www.ncbi.nlm nih.gov/pmc/articles/PMC2780141/ (accessed 11.05.2020).

5. Tenofsky PL, Beamer L, Smith RS. (2000). Ogilvie syndrome as a postoperative complication. Arch Surg, 2000;135(6):682-6. Available at: https://www.ncbi.nlm.nih. gov/pubmed/10843364/ (accessed 11.05.2020).

6. Remy P. (2012). Ogilvie Syndrome. NORD (National Organization for Rare Disorders). Available at: https:// rarediseases.org/rare-diseases/ogilvie-syndrome/ (accessed 11.05.2020).

7. Haj M, Haj M, Rockey DC. (2018). Ogilvie's syndrome: management and outcomes. Medicine, 2018;97(27):e11187. Available at: https://www.ncbi.nlm.nih.gov/pmc/articles/ PMC6076157/ (accessed 11.05.2020).

\title{
Post Covid-19 Guillain-Barre Syndrome: case report
}

\author{
Dr Robert Jones, MBBS, Foundation Year 2, FGH, Dr Hannah Kolbe, MBBS, GPST2, FGH \\ Dr Alan Barton, MB Bch, Consultant General Internal Medicine \& Geriatrics, FGH
}

\begin{abstract}
This is a case report of a 62-years-old male who developed neurological symptoms consistent with Guillain-Barre Syndrome following a hospital admission for Covid-19. As a new condition we feel there are learning points to be gained for clinicians managing patients recovering from Covid-19 and discuss the symptoms, signs, and management taken in this case. The patient thankfully made a full recovery, but his case is discussed in the context of other cases in the literature. We feel that this case is different and notable as it had a longer latent period between Covid-19 resolution and Guillain-Barre Syndrome onset than any other cases reported.
\end{abstract}

\section{HISTORY}

A 62-years-old male was brought to the Emergency Department by ambulance with a 3-day history of worsening weakness and sensory deficit of the upper and lower limbs.

On Saturday he had noted weakness and paraesthesia in his hands and feet. By Sunday, the weakness extended proximally in all four limbs so that he needed his wife's help to stand. By Monday he couldn't stand even with the help of his wife, and on Tuesday his sensory and motor symptoms in the hands and feet had worsened to a point where he contacted emergency services.

He had been recently discharged 15 days earlier following a 10 day inpatient stay for chronic obstructive pulmonary disease (COPD) exacerbation where he also tested positive for Covid-19 on admission. He had no associated diarrhoea on admission but had some diarrhoea whilst an inpatient. He had been recruited into the Recovery trial ritonavir arm. He recovered well and was discharged and had been well in the community.

$\mathrm{He}$ is a known patient of COPD, hypertension (HTN), obstructive sleep apnoea (OSA), using home continuous positive airway pressure (CPAP), and is on warfarin due to previous pulmonary embolism.

\section{EXAMINATION}

His upper limb power was reduced to grade 2 bilaterally at the shoulders, grade 3 at the elbows, and grade 4 wrist and hands. Lower limb power was grade 3 at the hips, 4 elsewhere. Sensation was reduced in the c6/7 dermatomes right arm and c6/7/8 left arm. In the lower limbs sensation was reduced in a glove and stocking fashion. He displayed areflexia even with reinforcement. Peak flow was 2101/ $\min$. 


\section{OBSERVATIONS}

Respiratory rate 21

Heart rate 101 regular

Temperature 36.8

Blood pressure 144/72

Saturations $94 \%$ OA

\section{INVESTIGATIONS}

His white cell count was 9.8 with lymphocytes 1.6. CRP was <6, B12 556, Folate 8, and CK 252.

An initial differential diagnosis included GuillainBarre Syndrome (GBS), intracranial pathology, and spinal cord compression.

A computed tomography (CT) scan of the head was undertaken showing widespread mild to moderate deep white matter and periventricular low attenuation deemed nonspecific on report.

\section{MANAGEMENT}

After initial assessment and investigations, he was referred to the medical team with a provisional diagnosis of GBS; they agreed for admission and neurology referral as an inpatient. He was admitted to the high dependency unit (HDU) with the intensive treatment unit (ITU) made aware in case respiratory effort decreased and there was a need for ventilation.

The next day he had a magnetic resonance image (MRI) of the spine taken reporting:

"Cervical spondylosis with degenerative disc disease. Compressive myelopathy at c5/6 and c6/7 levels causing cord indentation, secondary canal stenosis at c5/6 with no obvious cord oedema. Advise urgent neurosurgical referral."

Once the International normalised ratio was below 1.3 a lumbar puncture was performed showing:

- clear/colourless fluid

- protein 0.91

- glucose 4.1

- WCC 0 RBC 0 no organism seen on gram stain

The MRI results were discussed with the Preston Hospital neurosurgery team who advised that this was not likely to be causing the symptoms as there did not appear to be acute cord compression and that they would follow up as an outpatient once the GBS had resolved.

$\mathrm{He}$ was reviewed by our neurology consultant who agreed with GBS as the most likely cause, as the neurological symptoms had initially being peripheral and had progressed proximally and in severity. He had recently had a viral illness and cases of Covid-19 and similar viruses causing GBS are known about. Intravenous immunoglobulins ( $145 \mathrm{~g}$ over five days) treatment was started after finding a worsening of peak flow measurement.

After 4 days in hospital the ITU team changed to monitoring forced vital capacity, he was 2.5 litres, which was his normal value compared with 2 months prior to admission and it remained unchanged for the rest of his stay.

His muscle strength began to improve with treatment and by day seven he was stepped down to normal ward level care and then to our rehabilitation ward where he continued with physio and occupational therapy input.

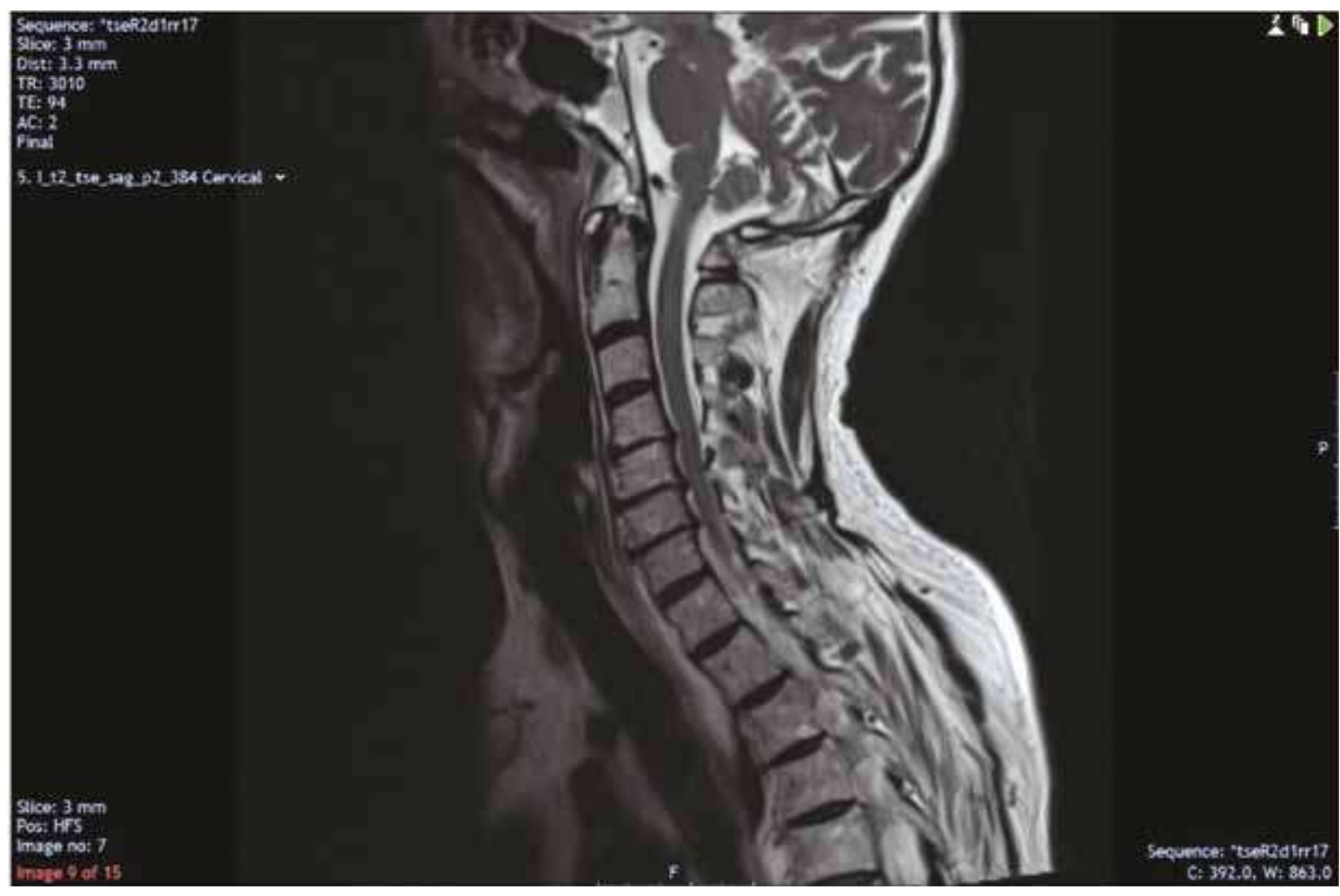

Figure 1: MRI of c-spine showing indentation of the cord at $\mathrm{c} 5 / 6$ and $\mathrm{c} 6 / 7$. 
Table 1: Peak flow measurements.

\begin{tabular}{|l|l|}
\hline Date & Peak flow (L/min) \\
\hline $5 / 5 / 20$ & 210 \\
\hline $6 / 5 / 20$ & 180 \\
\hline $7 / 5 / 20$ & 190 \\
\hline $8 / 5 / 20$ & 210 \\
\hline
\end{tabular}

\section{DISCUSSION}

Guillain-Barre syndrome is a disease of the nervous system. ${ }^{1}$ It is thought to be immune-related; whereby the body's natural defence mechanisms attack the nerve cells rather than the pathogens that enter the body, thus causing a wide range of problems.

Symptoms start peripherally, affecting mainly the hands and feet initially and working proximally. The initial symptoms can be numbness, weakness, and co-ordination problems with hands/feet, and over a short period things can get worse. In some cases issues can move centrally and affect breathing and swallowing.

Whilst most will make a full recovery, it is a serious condition that can be life-threatening and leave patients with a range of long-term problems such as chronic fatigue and paralysis. As in our case presented here, it is a condition that is more common in male adults. ${ }^{1}$

It is thought that the body's attack on nerve cells begins with the entry of a pathogen, which in turn triggers the defence mechanism. ${ }^{1}$ These pathogens can include respiratory viruses, and we will explore the published literature so far on the links between COVID-19 and GBS.

On 23rd January 2020 in China a female 62-yearold patient was admitted to hospital with GBS. ${ }^{2}$ Her blood test on admission showed lymphocytopenia and thrombocytopaenia, and she had recently travelled to the Wuhan region of China, although at this point she had none of the classic Covid-19 symptoms. On day eight she did develop a dry cough and fever and was tested for Covid-19, the test coming back positive. This case was different as GBS symptoms presented before Covid-19. She made a full recovery after 30 days. The authors of this case suggested that GBS could follow a different time course than the traditional post infectious GBS, with symptoms occurring alongside infection as has been reported with Zika virus. ${ }^{3}$

There have been reports of GBS symptoms presenting post Covid-19; five patients in Northern Italy were found to develop GBS symptoms within 5-10 days of Covid-19 symptoms onset. ${ }^{4}$ There has also been reported GBS cases in the past for related coronavirus illnesses such as Middle East Respiratory Syndrome. ${ }^{5}$

The American Journal of Neurology ${ }^{6}$ reports the case of a 71 year old male admitted to Accident and Emergency (A\&E) with paraesthesia of the lower limbs that rapidly evolved into flaccid tetraparesis. Prior to this he suffered with a fever for a few days, and scans on admission revealed a typical COVID-19 pneumonia and a swab result was positive. His symptoms of GBS developed before the symptoms of COVID-19 had passed, unlike the gentleman in our case. This raises a possible link to developing GBS and the severity of the COVID-19 infection - does a more severe case of COVID-19 (for example requiring hospital admission with oxygen therapy, non-invasive ventilation, or sedation and invasive ventilation) mean GBS is likely to present earlier?

Our case is the latest development of GBS following Covid-19 symptoms onset of these reports (25 days) and we hope that this case helps to add evidence to the potential for GBS development due to Covid-19 infection. More research needs to be conducted, following the discovery of Covid-19, into the risk factors for people subsequently developing GBS (is there a link to pre-existing medical conditions for example?) and are alternative treatment strategies needed when Covid-19 is responsible for the development of GBS.

\section{CONFLICTS OF INTEREST}

All authors would like to declare that they have no conflicts of interest when submitting this article.

Correspondence to:

Robert.jones1@mbht.nhs.uk Hannah.kolbe@mbht.nhs.uk Alan.barton@mbht.nhs.uk

\section{REFERENCES}

1. NHS (2020) Overview: Guillain-Barré syndrome. Available at: https://www.nhs.uk/conditions/guillain-barre-syndrome/ (accessed 23.10.2020).

2. Zhao H, Shen D, Zhou H, Liu J, Chen S. Guillain-Barre syndrome associated with SARS-CoV-2 infection: causality or coincidence? Lancet Neurology 2020;19(5):383384. Available at: https://www.thelancet.com/journals/ laneur/article/PIIS1474-4422(20)30109-5/fulltext (accessed 23.10.2020).

3. Brasil P, Sequira PC, Freitas AD, Zogbi HE, Calvet GA De Souza RV. Guillain-Barre syndrome associated with Zika virus infection. Lancet 2016;387(10026):1482. Available at: https://www.thelancet.com/journals/lancet/ article/PIIS0140-6736(16)30058-7/fulltext (accessed 23.10.2020)

4. Toscano G, Palmerini F, Ravaglia S et al. Guillain-Barre Syndrome associated with SARS-CoV-2. New England Journal of Medicine 2020;382:2574-2576. DOI: 10.1056/ NEJMc2009191. Available at: https://www.nejm.org/doi/ full/10.1056/NEJMc2009191 (accessed 23.10.2020).

5. Kim JE, Heo JH, Kim HO et al. Neurological complications during treatment of Middle East Respiratory Syndrome. Journal Clinical Neurology 2017;13(3):227-233. Available at: https://pubmed.ncbi.nlm.nih.gov/28748673/ (accessed 23.10.2020).

6. Alberti P, Beretta S, Piatti M et al. Guillain-Barré syndrome related to COVID-19 infection. American Academy of Neurology. 2020;7(4). Available at: https://nn.neurology. org/content/7/4/e741 (accessed 23.10.2020). 